\title{
Thank you to our Reviewers
}

Published online: 30 January 2021

(C) The Author(s), under exclusive licence to Springer-Verlag GmbH Germany, part of Springer Nature 2021, corrected publication 2021

The Editors of Structural and Multidisciplinary Optimization and the Publisher wish to thank the following reviewers, who have helped to maintain and improve the quality of the journal in 2020.

Gengdong Cheng and Ming Zhou

Editors-in-Chief

Balu A S

Niels Aage

Magd Abdel Wahab

Misganaw Abebe

Mohammad Abu-Zurayk

Osman ACAR

Erdem Acar

Wolfgang Achtziger

Frederico Afonso

Masoud Afrousheh

Seyed Saeed Ahmadisoleymani

Jaemyung Ahn

Hamid Akbarzadeh

Emre ALBAK

Alejandro Albanesi

Mustafa Al-Bazoon

Mazen Albazzan

Joe Alexandersen

Hugo Aliaga-Aguilar

Farbod Alijani

Douglas Allaire

Gregoire Allaire

Theodore Allen

James Allison

Sergio Almeida

Diego Alonso

Hugo Alvarez

Olaf Ambrozkiewicz

Oded Amir

Haichao An

Xuanyi An

Weigang An

G Ananthasuresh

Casper Andreasen

Erik Andreassen

Carla Tatiana Anflor
Cosmin Anitescu
Ruben Ansola
Carlos Antonio
Alejandro Aragón
Aurelio Araujo
Pedro Areias
Payam Asadi
Alireza Asadpoure
Gustavo Assis Da Silva
Vikrant Aute
Saeed Azad
Hideyuki Azegami
Aydin Azizi
Dario Azzimonti
Sangjune Bae
Seunghun Baek
Yingchun Bai
Aitor Baldomir
Ahmad Baroutaji
Jorge Barrera Cruz
Bruno Barroqueiro
Nathalie Bartoli
Ronald Bartz
Anirban Basudhar
Ali Asghar Bataleblu
Ahmed Bayoumy
Alparslan Bayrak
Pierre Beaurepaire
André Beck
Morad Behandish
Reza Behrou
Ashok Belegundu
José Bellido
Mohamed Amine Ben Abdallah
Poha

\author{
Malek BEN SALEM \\ Ernesto Benini \\ Martin Berggren \\ Dipankar Bhanja \\ Manav Bhatia \\ Soumya Bhattacharjya \\ Barron Bichon \\ Amin Bigham \\ Onur Bilgen \\ Mickael Binois \\ Rasmus Bjørk \\ Bartlomiej Blachowski \\ Bogdan Bochenek \\ Beniamin Bogosel \\ Sriman Pankaj Boindala \\ Karol Bołbotowski \\ Blaise Bourdin \\ Piotr Breitkopf \\ Benjamin Brelje \\ Neil W. Bressloff \\ Matteo Bruggi \\ Tinh Quoc Bui \\ Sujin Bureerat \\ Adrian Butscher \\ Shouyu CAI \\ Vítor Camacho \\ Felician Campean \\ Alfredo Canelas \\ Xiaojian Cao \\ Eduardo Cardoso \\ Hugo Carreira-Pedro \\ Josephine Carstensen \\ Aldemir Cavalini Jr \\ Rauno Cavallaro \\ Daniele Cerroni
}


Vincent Chabridon

Arunasis Chakraborty

Souvik Chakraborty

Souvik Chakraborty

Vivien Challis

Chia-Ming Chang

Navid Changizi

Anirban Chaudhuri

Payel Chaudhuri

Boyang Chen

Feifei Chen

Gaoxiang Chen

Hongquan Chen

Junwei Chen

Long Chen

Shikui Chen

Wei Chen

Weiqiu Chen

Wu-Yi Chen

Xianqi Chen

Xiaoqian Chen

Xueyan Chen

Yuhang Chen

Zhen Chen

Changqing Cheng

Chih-Chun Cheng

Gengdong Cheng

Jin Cheng

Jaskanwal Chhabra

Leonardo Chirco

Hyunkyoo Cho

Seonho Cho

Dong-Hoon Choi

Joo-Ho Choi

Myung-Jin Choi

Rajib Chowdhury

Jesper Christensen

Rasmus Christiansen

Junho Chun

Hayoung Chung

Miguel Cid Montoya

Pedro Coelho

Maxime Collet

Franco Concli

Simone Coniglio

Aleksander Czekanski

Daicong Da

Masoud Dahmardeh

Anna Dalklint

Thanh-Phong Dao

Charles Dapogny

Shuvodeep De

Breno De Almeida
Paulo De Castro

Daniel De Leon

Matthias De Lozzo

R. De Vries

Erin Decarlo

Ercan Dede

Arnoud Delissen

Henk Den Besten

Kongshu Deng

Shiguang Deng

Yongbo Deng

Boris Desmorat

Alejandro Diaz

Antonio Díaz-Álvarez

Robert Dienemann

Matteo Diez

Xiaohong Ding

Jaehyeok Doh

Hao-Wen Dong

Alberto Donoso

Alireza Doostan

Suguang Dou

Arinan Dourado

Christophe Droz

Bingxiao Du

Jianbin Du

Xianping Du

Xiaoping Du

Xiaosong Du

Zongliang Du

Libin Duan

Shuyong Duan

Sylvain Dubreuil

Fabian Duddeck

Peter Dunning

Maciej Dutkiewicz

Subhrajit Dutta

Pierre Duysinx

Grzegorz Dzierżanowski

Bassam El Said

Charbel-Pierre El Soueidy

Ali Elham

Helio Emmendoerfer

Tohid Erfani

Mohammadjavad Esfandiari

Pascal Etman

John Evans

Anton Evgrafov

Leandro Fadel Miguel

Nasim Fallahi

Zhirui Fan

Eduardo Fancello

Jianguang Fang
Hosam Fathy

Alex Feldestein

Florian Feppon

Scott Ferguson

David Fernandez

Eduardo Fernández

Felipe Fernandez Ayala

M. Giselle Fernandez-Godino

Federico Ferrari

Pedro Ferreira

Rafael Ferreira

Wallace Ferreira

Alex Ferrer

Eric FLORENTIN

Victor Franco Correia

Michael Friswell

Marco Fuentes-Huerta

Yoshiki Fukada

Arun Gain

Jean-François Gamache

Ranjan Ganguli

Tong Gao

Yunkai Gao

Andrea Garbo

Nicholas Gaul

Andrew Gaynor

Andrew Gaynor

Markus Geiss

Perle Geoffroy-Donders

Kazem Ghabraie

Ali Ghasemi

Seyede Fatemeh Ghoreishi

Sayan Ghosh

Frederic Gillot

Oliver Giraldo-Londoño

Sebastian Giusti

Massimiliano Gobbi

Tushar Goel

Christian Gogu

Guilherme Gomes

Wellison Gomes

Fernando Gomez

Juliano Gonçalves

Chunlin Gong

Recep M. Gorguluarslan

Hanno Gottschalk

Justin Gray

Jeroen Groen

Albert Groenwold

Xiaojun $\mathrm{Gu}$

Sofiane Guessasma

James Guest

Johann Guilleminot 
David Guirguis

Mehmet Guler

Ahmet Güllü

Tayfun Gundogdu

Qi Guo

Tinghao Guo

$\mathrm{Xu}$ Guo

Zhendong Guo

Saravana Kumar Gurunathan

Mariantonieta Gutierrez Soto

Seung-Hyun Ha

Raphael Haftka

Mohamed Hamdaoui

Karim Hamza

X. Han

Peng Hao

Helmut Harbrecht

Emadeldeen Hassan

Linwei He

Zheng He

Ping $\mathrm{He}$

Max Hendriks

Thibaut Hirschler

Van-Nam Hoang

Jie Hou

Shujuan Hou

Christopher Hoyle

Chao $\mathrm{Hu}$

Weifei $\mathrm{Hu}$

Zhangli $\mathrm{Hu}$

Zhen $\mathrm{Hu}$

Zhen $\mathrm{Hu}$

Zhengwei Hu

Daning Huang

Li Huang

Hai Huang

Xiaodong Huang

H.-Y. Hwang

Jaeyub Hyun

Hajime Igarashi

Teemu Ikonen

Mahdi Imani

Kevin Irick

Francois-Xavier Irisarri

Kazuhiro Izui

Kevin Jacobson

Lukas Jakabcin

Kai James

Hong-Lae Jang

Miche Jansen

Charles Jekel

Nicholas Jenkins

Kristian Jensen
Jakob Jensen

Chao Jiang

Long Jiang

Seung-Seop Jin

Zhao Jing

Soo-Ho Jo

Arun Johar

Philipp Junker

Sandilya Kambampati

Namwoo Kang

Young-Jin Kang

Zhan Kang

Yoshihiro Kanno

Levent Burak Kara

Ahsan Kareem

Ali Kaveh

Hesaneh Kazemi

Saeid Kazemzadeh Azad

Vahid Keshavarzzadeh

Vahid Keshavarzzadeh

Behrooz Keshtegar

Mohammad Khalid Jawed

Amar Khawaja

Il Yong Kim

Il Yong Kim

Nam Ho Kim

Yoonyoung Kim

Ravi Kishore

Satoshi Kitayama

Cesar Kiyono

Anders Klarbring

Vladimir Kobelev

Michal Kocvara

Nozomu KOGISO

Bonyong Koo

Stijn Koppen

Boris Kramer

Sergei Kucherenko

Robert J. Kuether

Mohammad Kurdi

Ricardo Lahuerta

Luciano Lamberti

Matthijs Langelaar

Antoine Laurain

Boyan Lazarov

Guenhael Le Quilliec

Dooho Lee

Guesuk Lee

Ikjin Lee

Jaehong Lee

Jaewook Lee

Jongsoo Lee

Sanghoon Lee
Tae Hee Lee

Yong Hoon Lee

Thierry Lefebvre

Fei Lei

Afonso Lemonge

Tomasz Lewiński

Gang Li

Guosong Li

Hao Li

Hongshuang Li

Lei Li

Lei $\mathrm{Li}$

Mian Li

Qing Li

Yu Li

Yumeng Li

Xuan Liang

Yuan Liang

Haitao Liao

Rhea Liem

Cicero Lima

Asger Limkilde

Esben Lindgaard

Chang Liu

Guiping Liu

Haitao Liu

$\mathrm{Hu}$ Liu

Hui Liu

Jie Liu

Jie Liu

Jikai Liu

Shaobin Liu

Shutian Liu

Tianchen Liu

Xiaomin Liu

Zhao Liu

Zhenyu Liu

Zhenyu Liu

Zhifeng Liu

Rafael Lopez

Zhenzhou Lu

Erik Lund

Jonas Lundgren

Quantian Luo

Yangjun Luo

Zhen Luo

Zhifan Luo

Nora Lüthen

Liye Lv

Matthew Lynch

Haitao Ma

Yongsheng Ma

Sankaran Mahadevan 
Laura Mainini

Milad Maleki

Rami Mansour

Jesús Martínez-Frutos

Joaquim Martins

Taiki Matsumura

Kurt Maute

Klaus Mecking

Andre Mendes

Joseba Mendiguren Olaeta

Zeng Meng

Günther Meschke

Georgios Michailidis

Amir Mirzendehdel

Samy Missoum

Florian Mitjana

Florian Mitjana

Wilfredo Montealegre Rubio

Marco Montemurro

Min-Yeong Moon

Ali Mortazavi

Maliki Moustapha

Chandan Mozumder

Samir Mulani

David Munk

Giuseppe Muscolino

Somanath Nagendra

Saideep Nannapaneni

Renato Nascimento

Omid Nejadseyfi

Miguel Neves

Tam Nguyen

Hung Nguyen-Xuan

Shinji Nishiwaki

Bin Niu

Cao NIU

Lise Noël

Yoojeong Noh

Yoojeong Noh

Tsuyoshi Nomura

Julián Norato

W.I. Notz

Antonio Novotny

Arian Novruzi

Hyunseok Oh

Makoto Ohsaki

José Luis Olazagoitia Olazagoitia

Jonathan Ollar

Max Opgenoord

Reinaldo Orselli

Ivan Oseledets

Carsten Othmer

Alberto Paganini
Francisco Palacios

Rafael Palacios

Pramudita Palar

Gyung-Jin Park

Amir Parnianifard

Jay Patel

Sachin Patil

Glaucio Paulino

Jakub Pawlicki

Claus Pedersen

Niels Pedersen

Daniel Peeters

Benjamin Peherstorfer

José Ricardo Pelaquim Mendes

Jeroen Pellens

Anderson Pereira

Nenad Petrovic

Lukas Pflug

Renato Picelli

Renato Picelli

Victor Picheny

Victor Picheny

Nicolò Pollini

Yannick Privat

Mariusz Pyrz

Sh Qian

Xiaoping Qian

Longhui Qin

Haobo Qiu

Zhiping Qiu

Domenico Quagliarella

Nestor Queipo

Carlos Quental

Amit R K

Timon Rabczuk

Timon Rabszuk

Palaniappan Ramu

Pritam Ranjan

Mostafa Ranjbar

Steven Reagan

Jean Rodolphe Roche

Helder Rodrigues

Rene Rodriguez

Fabio Roli

David Romero

Vicente J. Romero

Juan Romero

Jordi Romeu Garbí

Shilun Ruan

Martin Ruess

Markus Rumpfkeil

Mehmet Saka

Ruben Andres Salas Varela
Miguel Salazar De Troya

Leandro Salviano

Yuki Sato

Roger Sauer

Marco Savoia

Kiichiro Sawada

Anupam Saxena

Mark Schenk

Mattias Schevenels

Martin Pierre Schmidt

Axel Schumacher

Jaco Schutte

David Seifert

Abdollah Shafieezadeh

Mohammad Shahabsafa

Mohammad Shahabsafa

Sunil Kumar Sharma

Deepak Sharma

Masatoshi Shimoda

Jaekwan Shin

Martin Siebenborn

Ole Sigmund

Amilton Silva

Emilio Silva

Olavo Silva

Hemant Singh

Prem Singh

Raghavendra Sivapuram

Douglas Smith

Tomasz Sokół

Kai Song

Xueguan Song

Fazil Sonmez

Johannes Sperber

Vm Sreehari

Balaji Srinivasan

Nielen Stander

Bret Stanford

Tino Stankovic

Georgios Stavroulakis

Michael Stingl

Mathias Stolpe

Niclas Strömberg

Eric Sturler

Kevin Sturm

Karthick Subramaniam

Afzal Suleman

Pierre Sullivan

Beibei Sun

Jialiang Sun

Kangkang Sun

Zhi Sun

Alok Sutradhar 
Laura Swiler

Hossein Taghipoor

Meisam Takalloozadeh

Akihiro Takezawa

Jun Hui Tam

Jun Tao

Rouhollah Tavakoli

Rodrigo Tavares

Ghanshyam Tejani

Kenjiro Terada

Mishal Thapa

Carl-Johan Thore

Kuo Tian

Kuo Tian

Albert To

Liyong Tong

Mazdak Tootkaboni

André Torii

Francesco Tornabene

Daniel Tortorelli

Andres Tovar

Anh Tran

Vinay Ummidivarapu

Ramakrishna Uppari

Emiel Van De Ven

Fred Van Keulen

Gieljan Vantyghem

Krishna Veluru

Kumar Vemaganti

Paolo Venini

Gerhard Venter

Natasha Vermaak

Felipe Viana

William Vicente

Victor Victor Fachinotti

Lara Vieira

Manav Vohra

Ernani Volpe

Eddie Wadbro

Mathias Wallin

Bo Wang

Bo Ping Wang

Charlie Wang

Chong Wang

Chuang Wang

Dengfeng Wang

Fengwen Wang

G. Gary Wang

Haoyu Wang

$\mathrm{Hu}$ Wang

Jian Wang

Lei Wang
Liwei Wang

Liwei Wang

Michael Wang

Peng Wang

Pingfeng Wang

Qian Wang

Weiming Wang

Xiaojun Wang

Xuan Wang

Yiqiang Wang

Zequn Wang

Zhenpei Wang

Zhonglai Wang

Seth Watts

Peng Wei

Pengfei Wei

Xinpeng Wei

Justin Weibel

Fabian Wein

Daniel White

Chi Wu

Di Wu

Di Wu

Jinglai Wu

Jun Wu

Yizhong Wu

Zhimin Xi

Liang Xia

Qi XIA

Mi Xiao

Fenfen Xiong

Hongyi Xu

Jun Xu

Liang Xu

Xian Xu

Guofeng Xue

Kentaro Yaji

Takayuki YAMADA

Kun Yan

Jun Yan

Cheng Yang

Dixiong Yang

Kai Yang

Kaike Yang

Ren-Jye Yang

Yang Yang

Wen Yao

Wenjing Ye

Alden Yellowhorse

Ping $\mathrm{Yi}$

Kazuo Yonekura

Byeng Youn
Sung-Kie Youn

Minghao $\mathrm{Yu}$

Yang Yu

Yonggyun $\mathrm{Yu}$

Yigit Yucesan

Kyeong-Soo Yun

Mohammed Amine Zafrane

Ihor Zanevskyy

Tomas Zegard

Samih Zein

Zhi Zeng

Bin Zhang

Dequan Zhang

Fan Zhang

Guodong Zhang

Jingyao ZHANG

Lei Zhang

Ruifu Zhang

Shanglong Zhang

Tianxiao Zhang

W. H. Zhang

Weisheng Zhang

Xiaojia Zhang

Xufang Zhang

Yiming Zhang

Yong Zhang

Yongcun Zhang

Zhao Zhang

Zhengui Zhang

Guozhong Zhao

Ling Zhao

Min Zhao

Wanzhong Zhao

Jing Zheng

Jun Zheng

Kai Zheng

Huang Zhengdong

Changting Zhong

Kun Zhou

Ming Zhou

Mingdong Zhou

Pingzhang Zhou

Qi Zhou

Yuqing Zhou

Jianxiong Zhu

Ji-Hong Zhu

Yichao Zhu

Markus Zimmermann

Jun Zou

Wenjie Zuo 
